\title{
A Cross-Generational Study of Contraception and Reproductive Health Among Sudanese and Eritrean Women in Brisbane, Australia
}

\begin{abstract}
This study conducted in Brisbane, Australia, was undertaken with a cross-section of Sudanese and Eritrean mothers and daughters. We explored and documented the women's intergenerational experiences and knowledge of reproductive health and contraception. Underpinned by a qualitative approach, focus group discussions were undertaken along with key informant interviews with health and multicultural sector professionals. Through examination of knowledge shared, the analysis distilled key aspects of intergenerational fears, cultural safety, and health. Participants proposed recommendations on how refugee and migrant women in Australia and resettled countries globally can more effectively and holistically exercise their sexual and reproductive health (SRH) rights.
\end{abstract}

The United Nations High Commissioner for Refugees (UNHCR) reported that by the end of 2012, globally, there were approximately 10.5 million people considered to be refugees or to to be in a refugee-like situation (UNHCR, 2013). By the conclusion of 2012, developing countries including Pakistan, Iran, Kenya, Ethiopia and Jordan, were reported to host 81 per cent ( 8.5 million) of the global refugee population (UNHCR, 2013). The developed countries hosting the largest numbers of resettled refugees in 2012 included North America (the United States of America and Canada) who hosted the resettlement of more than 60,000 refugees and Australia, who increased its UNHCRreferred resettlement places to 12,000 (within its overall annual humanitarian intake of 20,000 persons) (UNHCR, 2013). Asylum application rates in many European countries increased in 2012 with the Nordic countries receiving nearly 63,000 asylum applications (the majority in Sweden) and Germany accounting for the highest number of new applications $(64,500)$ (UNHCR, 2013). With the global refugee and migrant populations resettling in countries and cultures vastly different from their own, it is imperative that host countries be able to address the specific health care needs 
of these new and vulnerable members of society (Benson \& Smith, 2007; Henderson \& Kendall, 2011)

In 2011, the Australia government estimated that almost 6 million migrants, born in over 200 countries and speaking over 400 different languages, currently live in Australia (Australian Bureau of Statistics [ABS], 2011). The scope of the Australian Humanitarian Resettlement Program, facilitated by the Department of Immigration and Citizenship (DIAC), is subject to: the United Nations Commissioner for Refugees (UNHCR) assessments of the resettlement needs of refugees; community consultations with the Minister for Immigration and Citizenship; and Australia's capacity to support the humanitarian resettlement programme (DIAC, 2011).

From 1997 to 2007, the five African countries and regions accounting for the most settler arrivals in Australia under the Humanitarian Program were Sudan $(22,445)$, Other Central and West Africa (3,796), Ethiopia (2,714), Sierra Leone $(2,477)$ and Somalia $(2,373)$ (ABS, 2008). During 2001 - 2007 over 5,000 African refugees resettled in the state of Queensland, located on the east coast of Australia (Harte, Childs, \& Hastings, 2011; Queensland Government, 2008). The vast majority of these individuals settled in Brisbane, the capital city, which as of June 2011, had a population of 1,079,392 (Brisbane City Council, 2012; Harte et al., 2011; Murray, Windsor, Parker, \& Tewfik, 2010; Queensland Government, 2008). With the growing number of African refugees and migrants resettling in Brisbane, there is an increased need for the specific health care requirements of these communities to be addressed (Benson \& Smith, 2007; Henderson \& Kendall, 2011). It was for this reason we sought to document and examine the SRH experiences and knowledge of women from Sudanese and Eritrean backgrounds. The implications of our research have the potential to not only influence health care provision and policy for culturally and linguistically diverse (CALD) women in Brisbane, but are also of relevance globally in other countries where refugee and migrants seek to resettle.

\section{LITERATURE REVIEW}

Culturally and Linguistically Diverse (CALD) Communities 
Cultural and linguistic diversity in Australian communities is increasing with $22 \%$ of the population being born in a foreign country (DIAC, 2007; Thomas et al., 2010). The term CALD describes a population of people, including refugees (both self-identified and born in refugeesource countries), who are non-English speaking, may use interpreters and men, women and children born outside of Australia (Thomas, Beckmann \& Gibbons, 2010).

Disparities exist in the provision of health services to ethnic and racial minority groups (Carroll, Epsein, Fiscella, Volpe, Diax \& Omar, 2007; Henderson \& Kendall, 2011; Kelaher, Williams \& Manderson, 1999; Thomas et al., 2010). Language and/or cultural and/or religious barriers may obstruct ability or willingness to seek and/or understand reproductive health advice (Carroll et al., 2007; Henderson, Kendal and See, 2011; Thomas et al., 2010). Thomas, Beckmann and Gibbons (2010), in their study on the effect of cultural and linguistic diversity on pregnancy outcomes, found that in Australia, education of health care professionals around unique cultural issues relating to reproductive health is essential for the highest level of care provision for women in CALD communities.

Strategies that reduce language, cultural, religious and economic barriers to health services and information for CALD communities are vital to improving their health status (Henderson \& Kendall, 2011; Henderson et al., 2011; Kelaher et al., 1999). Henderson and Kendall (2011) found, in their study in Logan, Queensland, that Sudanese participants reported that their health beliefs, cultural values and traditional health treatments are often overlooked by Australian health professionals. Participants also reported that they were not adequately informed on the cultural differences between their country of origin and the Australian health care system (Henderson \& Kendall, 2011). The lack of information was a key determinant of dissatisfaction among study participants regarding their treatment by health care professionals, and subsequent apprehension to contact health care providers (Henderson \& Kendall, 2011).

Benson and Smith (2007) state that refugees and General Practitioners (GPs) are often confronted with unfamiliar cultural differences that impact on both the patients' and medical professionals' experience. Unfamiliar referral hierarchy, health literacy, and body language, as well as attitudes towards medication and treatment expectations, create barriers to health care seeking 
and the adherence to treatment regimens (Benson \& Smith, 2007; Henderson \& Kendall, 2011). The progression from initial health assessments of refugees upon their arrival into Australia by specialised health units, to the current contracts with the Department of Immigration and Citizenship (DIAC), continues to create multifaceted challenges for both patients and health care professionals (Benson \& Smith, 2007; Henderson \& Kendall, 2011). The current process of encouraging community GPs' involvement as first contact for care reiterates the need for health care professionals to be culturally sensitive to the specific health care needs of the refugee and migrant community (Benson \& Smith, 2007; Henderson \& Kendall 2011).

\section{Reproductive Health and Contraception Knowledge}

In 1995, the United Nations Fourth World Conference on Women, held in Beijing, China, recognised that deeply entrenched social and cultural discrimination creates major barriers to the achievement of reproductive health for women especially from the developing world (United Nations Population Fund [UNFPA], 2011). Women from disadvantaged communities often experience barriers in obtaining reproductive health and contraception knowledge and also experience problems accessing health care facilities (UNFPA, 2011; World Health Organization [WHO], 2011).

Carroll, Epstein, Fiscella, Volpe, Diaz, \& Omar's (2007) study on knowledge and beliefs about health promotion and preventive health care among Somali refugee women living in the United States, found that traditional remedies and rituals, the role of religion, and access to health care facilities play an important role in health care beliefs and knowledge. Researchers have highlighted the link between religious and sociocultural characteristics to health status and health care seeking activities (Carroll et al., 2007; Henderson et al., 2011, UNFPA, 2011). Socioeconomic status and education are frequently documented as key determinants of health outcomes, particularly in women (WHO, 2010).

\section{The Empowerment of Women}


The empowerment of women through education within formal schooling, as well as grassroots SRH education, has been shown to have an impact on maternal and child mortality and morbidity in developing countries (Implementing Best Practice Initiative [IPB], 2010; WHO, 2008, 2011). The World Health Organization (WHO) states that empowering women to take control of their reproductive health can assist them to space and limit pregnancies, directly impacting their health and the well-being of their family, as well as the outcome of each pregnancy (WHO, 2011). It is estimated that about 200 million couples in developing countries would like to stop or delay childbearing but are unable to access any form of contraception (WHO, 2011). Barriers to contraception use include: limited choice of methods; limited access (due to age, marital status, cultural and socio-economic factors); fear of side effects; poor quality of services; gender based barriers; and religious reasons and often continue to inhibit women's reproductive health rights in their countries of resettlement (IPB, 2010; Murray et al., 2010; WHO, 2008, 2011). Barriers to positive SRH care seeking attitudes and contraception use must be understood by service providers to enable greater reproductive health outcomes for these women (Murray et al., 2010; Henderson et al., 2011).

\section{STUDY DESIGN}

Due to the sensitive nature of this study, and taking into account issues of religion, culture and language barriers, a qualitative research approach was used. Utilising qualitative research methods such as focus group discussions (FGDs) and in-depth interviews (IDIs), perceptions, feelings and experiences relating to reproductive health and contraception of a cross-section of Sudanese and Eritrean women were explored. The qualitative research design also permitted for a rich understanding and in-depth exploration of the health needs of the target group (Jirojwong \& Liamputtong, 2009). Ruppenthal, Tuck and Gagnon (2005) argue that the emotional support displayed between group members during a FGD is one of the key assets of this research method. The oral tradition amongst indigenous groups also supports the FGD methodology as a means of gathering information (Ruppenthal et al., 2005). 
The aim of our study was to explore, document and examine the intergenerational experiences and knowledge of reproductive health and contraception a cross-section of Sudanese and Eritrean migrant and refugee mothers and daughters currently living in Brisbane, Australia. The research was carried out in conjunction with a University in Western Australia and a not-forprofit Organisation working in the area of Sexual and Reproductive Health (SRH) in Queensland.

\section{The Conceptual Framework Used for the Study}

The Psychosocial Framework (2000) developed by the Psychosocial Working Group (PWG) was the conceptual framework that underpinned the study. The psychosocial well-being of an individual is defined in respect to three core domains: human capacity; social ecology; and culture and values (PWG, 2003). The Psychosocial Framework is particularly applicable to research involving vulnerable populations from post-conflict nations and was therefore chosen to inform the proposed research and guide the analysis (PWG, 2003).

\section{METHODS}

\section{Participant Recruitment Strategies}

The Sudanese and Eritrean communities were chosen for this research as they are communities that have existing ties with the SRH Organisation involved in the research and form a large proportion of the refugee and migrant population in Brisbane (ABS, 2008; Queensland Government, 2008). These connections enabled access to community leaders who assisted in the participant recruitment process. Snowballing and purposive sampling was predominantly used to recruit participants (Burns, 2000; Hinton \& Earnest, 2009).

The mothers' focus group discussion was held with eight participants and the daughters' group had a total of five participants. Inclusion criteria for research participation was: being female from a Sudanese or Eritrean migrant or refugee background; aged between 35-55 years or 18-30 years; currently live in Brisbane; have the capacity to give informed consent; and the ability to speak and understand English. 


\section{Focus Group Discussions (FGDs)}

The FGDs were differentiated by two age groups, a mothers' group (35-55 years of age) and a daughters' group (18-30 years of age). Conducting separate FGDs for the different generations of women enabled a comparison of intergenerational experiences, knowledge and opinions about contraception and reproductive health. Cultural and religious similarities between the two groups assisted cohesion and positive group dynamic as well as the creation of a safe and comfortable environment conducive to dialogue (Mengisteab, 2010). Questions were piloted on a small group before the FGDs were conducted and feedback incorporated to refine questions and themes to ensure participant understanding and cultural sensitivity (Henderson \& Kendall, 2011).

Before the commencement of the FGDs, participants and the research team shared a meal together to help establish rapport. Open-ended questions, sensitive to the context, were used to facilitate dialogue between participants and to generate diversity of views (Hinton \& Earnest, 2009; Ruppenthal et al., 2005). Key themes included contraception and reproductive health knowledge; access and barriers to contraception and reproductive health knowledge and services; and how SRH services can improve to increase uptake. Although participants were able to speak and understand English, two trained interpreters (fluent in Arabic and Tigrinya) were present during the mothers' FGD to enable participants to express themselves in their own language if they felt the need to. It also helped to minimise language barriers and ensure that participants had a thorough understanding of topics being discussed (Henderson \& Kendall, 2011).

\section{In-Depth Interviews (IDIs) with Key Informants}

Two IDIs, conducted with a Multicultural Women's Health Project Coordinator and a SRH Advanced Practice Nurse, were a valuable tool for collecting information relating to SRH and contraception issues specifically concerning the target population (Jirojwong \& Liamputtong, 2009). The information gathered during the IDIs was used to cross check data collected from both FGDs and compared with current literature (Minichiello, 2008; Strohschein, Merry, Thomas \& Gagnon, 2010) 


\section{Quality Criteria}

Throughout the research process, an audit trail ensured that information relating to the study context, research methodology and data analysis were documented to permit transparency of the research as well as enabling research replication (Hinton \& Earnest, 2010; Rodgers \& Cowles, 1993; Sharts-Hopko, 2002). Member checking, the on-going process of sharing findings with participants to ensure accuracy of data, also took place as well as a systematic review of relevant and recent literature (Hinton \& Earnest, 2010; Sharts-Hopko, 2002).

\section{Ethical Considerations}

Ethical approval to conduct this study was obtained from the SRH Organisation and University involved. The research also utilised the National Health and Medical Research Council's (NHMRC) National Statement on Ethical Conduct in Human Research as an ethical standard of practice framework (NHMRC, 2007). All participants were recruited on a voluntary basis for the purpose of this study and, to ensure anonymity, pseudonyms have been used (NHMRC, 2007). Information about the research was provided to participants to ensure adequate understanding of research aims (NHMRC, 2007). Oral or written informed consent was obtained depending on participant preference (Hinton \& Earnest, 2010). Before commencement of the research, participants were informed of their right to withdraw from the research process at any time (NHMRC, 2007). To ensure confidentiality, all research data is stored in a secure location with access only available to the first author (NHMRC, 2007). In appreciation of their involvement and time, participants were given a small gift, refreshments were provided and certificates of participation offered. The results and the research report were also shared with participants and the organisation.

\footnotetext{
ANALYSIS

After completion of FGDs and IDIs, audio-recordings of the sessions were transcribed verbatim for analysis (Hinton \& Earnest, 2010). The transcripts were read and re-read by the principal researcher and also by a cultural interpreter to ensure cultural understanding and meaning
} 
of data was accurately interpreted and ensured the integrity and reliability of the data (Burns, 2000; Hinton \& Earnest, 2010; Jirojwong \& Liamputtong, 2009). A thematic content analysis was then undertaken to collate and condense the information gathered into specific themes (Joyce, Earnest, De Mori \& Silvagni, 2010; Mohajer, Bessarab \& Earnest, 2009). Internal validity, the process of exploring alternative explanations for observed events, was enhanced by member checks with participants (Hinton \& Earnest, 2010). Researchers have documented that culture, religion, education and migration experiences affect participants' perceptions of questions in health research and it was therefore essential that key themes were interpreted accurately (Hinton \& Earnest, 2010; Strohschein et al., 2010).

Distinct themes were refined from the thematic content analysis of the FGDs and IDIs and participant proposed recommendations were used to inform proposed policy and practice recommendations. While we were careful to retain the integrity of participants' voices, minor grammatical edits were made to participant quotes (Hebbani, Obijiofor \& Bristed, 2009). To ensure cultural understanding and meaning was accurately interpreted, the thematic interpretations were validated by a cultural interpreter and member checking of comments by study participants was concurrently undertaken (Hinton \& Earnest, 2010).

\section{FINDINGS PART 1: FOCUS GROUP DISCUSSIONS}

\section{Contraceptive Knowledge}

Themes relating to contraception knowledge that emerged from the FGDs centred on the use of contraception to: prevent pregnancy; stop transmission of sexually transmitted infections (STIs); assist with child spacing; and control menstrual cycles. Participants in both mothers' and daughters' groups reported varying levels of knowledge relating to the types of contraception available, however, both groups demonstrated a good understanding of why individuals use contraception. Several participants in both FGDs were able to identify the majority of contraceptives currently available, with participants in the daughters' group also mentioning the contraceptive patch (not currently available in Queensland). While no participant identified vaginal rings) or diaphragms as contraceptive options by name, participants in the daughters' FGD 
demonstrated awareness of these options. Both groups demonstrated knowledge about condoms as a contraceptive option and as a form of prevention against STIs, however, the female condom was not specifically mentioned. Comparatively, the daughters' group expressed more peer-related discussion about contraception than the mothers' group. The two age groups also focused on different aspects of contraception use highlighting the key factors behind its use. Themes of protection against STIs were more prominent in the daughters' group compared with the mothers'.

I guess number one to prevent pregnancy but secondly, which is more on the condom side, is to prevent STIs and STDs so those are the two main reasons I can think of why a lot of people would use contraceptives. (Lilly, daughters' FGD)

The use of contraception to control birth spacing was a recurring theme within the mothers' FGD and it was also highlighted that this is an important factor in enabling a healthy family environment. Participants in the mothers' FGD also reported the positive impact contraception use has on a woman's health, Mahea, shared: 'Contraception is used to stop the lady from becoming pregnant. It keeps her healthy. If the lady is pregnant every year, it is not healthy for her'. Another emerging theme from the mothers' FGD was the use of contraception, particularly the oral contraceptive pill, to manage heavy or difficult menstrual cycles, Erteri explained: 'Especially when you move to different country your period can be very heavy and irregular so I use to take the pill for that irregular bleeding'. While participants in the daughters' FGD reported the occurrence of painful or difficult periods, this was not detailed to be a key reason behind contraceptive use.

Participants in the daughters' FGD also reported the difficulties faced by young people within their community in understanding the importance of contraception, how to use it, and the reasons behind its use.

A lot of our people they are not educated about these things and honestly they are hard to grasp, especially when sex just happens once in a while. They find it really hard to understand and hard to work contraception and that scares some people. (Lilly, daughters' $F G D)$ 


\section{Access to Contraception and Reproductive Health Care Services}

Recurring themes relating to the access of contraception at medical clinics, sexual health clinics, family planning services, supermarkets and pharmacies were common within both mothers' and daughters' FGDs. Participants in the daughters' group also reported on the confidentiality issues young people face when attending health care clinics and how this can deter young people from accessing contraceptive and reproductive health services.

It's a scary thing and young people don't want older people to know their business especially about sex because it's supposed to be taboo in our culture. (Lilly, daughters' FGD)

\section{Barriers to Contraception Use and SRH Knowledge and Services}

Both groups demonstrated insights into barriers faced by people within their communities wanting to access contraception and SRH services. Sociocultural barriers were expressed as a dominant theme with cultural competency issues at health care clinics and language barriers reported. Participants also expressed concerns about contraception use, lack of communication within the family environment about SRH as well as male and female dynamics as barriers to contraception and reproductive health care.

\section{Socio-cultural barriers}

Cultural issues relating to the taboo nature of reproductive and sexual health issues in both Sudanese and Eritrean cultures was a predominant and recurring theme throughout both mothers' and daughters' FGDs.

They feel ashamed about talking about sex. They have problems using names like vagina, penis and the rest of it. They think it's really a big issue that cannot ever be forgiven so it's really hard for them to speak about having STDs. (Simo, mothers' FGD) 
Cultural issues relating to health care provision and cultural competency of health care professionals was also discussed during the daughters' FGD.

Not knowing where to find services and, not knowing where to find a doctor you can trust is a problem for people. A lot of the African people, like some of the Sudanese people, they're not comfortable going to a white persons clinic. When the patient starts to ask the doctor questions, they just think 'well they won't understand what I believe in because they always bring in what they believe'. They're not comfortable talking to someone who they assume is not going to have any idea about their culture. (Lilly, daughters' FGD)

\section{Language barriers}

Language barriers were a dominant and recurring theme in both mothers' and daughters' FGDs. Lilly, a participant in the daughters' FGD commented that 'sometimes it's hard to understand the way the doctors explain it. Language barriers are a huge issue'. Gender issues and the use of male interpreters were also noted as inhibitors of contraception and reproductive health knowledge and access to services by both generational groups. Sara, a participant in the daughters' FGD, noted that 'some women maybe wouldn't talk because they won't get interpreters in some of the GP places or they have a male $G P^{\prime}$. Being able to communicate comfortably and openly to health care providers was noted as an important factor for group members in the provision of effective and culturally sensitive health care.

\section{Concerns about contraceptive use}

Concerns relating to side effects of contraceptive use were a recurring theme within both mothers' and daughters' FGD. Weight gain, irregular or heavy bleeding, fertility issues, skin breakouts, lethargy, as well as remembering to consistently take contraception, were reported as concerns. Tango, a participant in the mothers' FGD spoke about her concerns with the side effects of contraception use 'putting on weight was worrying me'. Other issues such as disruption to an individual's normal menstrual cycle were also noted as concerns. While many of the participants shared their own experiences of having used a contraceptive that did not suit them physically 
and/or psychologically, participants noted they were able to change contraception methods until they found one that worked well for them.

I think people like to use Implanon but they get a bit of scared because they won't have a period. Then they will say 'oh no if I don't have a period then I better have the pill or the injection'. (Sara, daughters' FGD)

Participants in both mothers' and daughters' FGDs also reported concerns about the effectiveness of contraception use, in particular the oral contraceptive pill. Experiences of falling pregnant while using the oral contraceptive pill were shared by two members of the mothers' FGD. Although concerns regarding effectiveness of contraception or side effects from contraceptive use were shared, none of the participants stated these as deterrents from utilising contraception all together.

I used to take the pill for irregular bleeding. I heard some people say if you want another child you can't get pregnant easily if you take the pill but I found out when I was taking the pill that I was pregnant with my daughter, so they did not work. (Erteri, mothers' FGD)

\section{Lack of discussion about SRH within the family environment}

Members of the daughters' group specifically mentioned the lack of communication within the family especially between mothers and daughters about SRH. Suzy commented on the lack of awareness some parents have about their children's sexual activities: 'They assume their daughters' are not sexually active. They don't really know what their kids are doing out there'. Parental influence on beliefs young people have regarding SRH, and the choices they make relating to contraception use, was a key theme in both mothers' and daughters' FGDs.

It's not just educating the young people, if they can educate the parents I think that will help a lot because the parents influence their kids pretty much in everything. The problem in our culture is the parents are not really talking to their kids about these things. The young people don't want to use contraception because their parents are probably not saying anything to them. There's always that confusion, which side to take. The school side (SRH education) or what the parents are saying or what the parents are not saying. It confuses a lot of young 
people and when the young person is confused they just do whatever they want. (Lilly, daughters' FGD)

Several of the mothers' FGD participants also spoke about their experiences going through puberty and how discussion within the family about puberty and reproductive health is difficult or in most cases non-existent. Azahar, a participant in the mothers' FGD stated: 'I have been married 16 years and didn't know much about these things (reproductive health). I've learned through experience, not with checking with somebody'. Cultural issues surrounding the taboos of SRH in Eritrean and Sudanese cultures were highlighted as barriers to discussion.

In our culture we don't talk about sexual health or reproductive health. When I got my period I didn't know that it was something natural happening so I asked my Aunty. She said that it's normal when the girl comes to certain age. That's all I learned about reproductive health. The rest I learned through marriage. (Ahalam, mothers' FGD)

\section{Male and female dynamics and the use of contraception}

Participants from both age groups commented on the dynamics between males and females regarding the use of contraception. Participants in the mothers' FGD stated that for effective use of contraception to occur, both the man and the women need to be in open discussion about its use. Tango, a participant in the mothers' FGD commented: 'to use contraception it has to be an agreement between the wife and the husband'. It was noted that this can be difficult as some men within their culture object to the use of contraception.

There are some men that object to contraception, they say 'no you're not going to take it'. You can't hide taking it from your husband, so it has to be clear and open between the two partners. (Simo, mothers' FGD)

Participants in the daughters' FGD also noted that the male/female dynamic can put pressure on women and inhibit their reproductive health rights. 
We've grown up in a culture where our parents didn't use them so the boys think 'I'm not going to use a condom' so that is a huge problem, because the girls don't want lose the boys as its very high competition nowadays. The girls will do anything to keep their boys and that means they will go without protection. (Lilly, daughters' FGD)

Research participants in the mothers' FGD reported on the importance of SRH education for men as well as women and commented on barriers that can inhibit education.

If it's a female giving health education to men, they look a little bit negatively on it. There should be health education not only for women, but men and women. Separate groups need to be held for men and women otherwise they will be too shy to talk. There should be a male staff member who can look after the men because they're really negative about family planning, their objective is 'I've married you to have babies'. (Simo, mothers' FGD)

\section{Misconception about sexually transmitted diseases}

A recurring theme detailed in the mothers' FGD relates to misconceptions many newly arrived refugees and migrants have about sexually transmitted disease prevalence in Australia. Participants stated that many young people within their communities believed that HIV/AIDS and STIs do not exist in Australia and as a result, are not practicing safe sexual behaviours. Participants in the daughters' FGD reported risky sexual behaviours, such as unprotected sex, as common amongst the younger generation. Lack of knowledge about safe sexual practices was stated to be compounded by the sociocultural barriers inhibiting contraception use and SRH service utilisation.

Because you have to pass a health examination when you come to Australia, people, both adults and youths, have the misunderstanding that in Australia there are no diseases. So they practice unsafe sex and then they get diseases. (Makda, mothers' FGD)

\section{How Health Services Can Improve: Meeting the communities' needs}

Participants were asked their views on how services could improve to better meet their SRH needs. Makda, a participant in the mothers' FGD shared: 'give continual education about health issues 
and about how important it is in the lives of not only the woman but the whole family, the man and the children as well'. Community and whole family involvement was a dominant and recurring theme in the participants' opinions regarding effective SRH education and services.

Contacting the leaders in the communities, I think that would help a lot because they're the ones who can distribute the information and they will know how to do it because they know their people. (Lilly, daughters' FGD)

Participants in both the mothers' and daughters' FGD highlighted the importance of creating a safe environment where people feel comfortable to access services and discuss health concerns. It was also reported that small gatherings would be more effective than large group educational sessions. If groups could be organised to give them the message about reproductive health it would be good. Most of the girls from our cultural background don't know about their bodies. So I feel that it is good if we be able to organise small groups for those discussions then they are able to give them the message and they would also be future leaders that can teach others. (Tango, mothers' FGD)

Cultural sensitivity and addressing language barriers were also key themes, with participants highlighting the importance that implementations and services must be culturally sensitive for effective SRH education to occur. Makda, a participant in the mothers' FGD highlighted this need: 'Provide them information in a culturally sensitive way. Provide them with interpreters.' Providing accessible, regular and culturally sensitive SRH education was a dominant and recurring theme throughout both mothers' and daughters' FGD.

Services that are culturally sensitive or appropriate will be very, very helpful. In regard to Sudanese people in particular, I don't think it's easy for them to access health care services because I don't even think they know those kinds of services exist. Also, I don't think they believe in them so education is needed. (Lilly, daughters' FGD) 
Two key informants were purposively sampled for their specific and diverse experiences in working with women from CALD backgrounds, particularly in the area of SRH. The key informant contacts were a Multicultural Women's Health Project Coordinator and an Advanced Practice Nurse, who specialises in women's health and family planning.

\section{Contraception and SRH Education}

SRH education, through both formal schooling and grassroots education, plays a key factor in the utilisation of contraception and SRH care services of women from CALD backgrounds and was a recurring theme in the IDIs.

We try to make SRH knowledge accessible to women by addressing cultural issues and language barriers. It is important that our health care workers are able to communicate with women in their own languages and come from a similar cultural background. This way, SRH knowledge can more effectively be disseminated. (Multicultural Women's Health Project Coordinator)

\section{Barriers to Service Utilisation and Contraception Use}

Culture and religion can be important factors in a woman's decision to access contraception and were noted as key barriers in the use of contraception and the utilisation of SRH services.

The cultural and religious beliefs for many different communities and countries in Africa include the strong belief that children are a gift from God. It is therefore the belief of many people that the use of contraception goes against religious beliefs and values and can be a barrier to its use. (Multicultural Women's Health Project Coordinator)

Financial barriers to SRH care services were also noted as an inhibiting factor in women's utilisation of services.

There is often limited or no SRH services in many of their countries of origin. If they are available, they are often expensive. When they arrive in Australia they often assume it is the same here. (Multicultural Women's Health Project Coordinator) 
It was stated that even when women from CALD backgrounds know a service is free, they place little priority on SRH, particularly if they have migrated from war-torn and impoverished communities. Cost of transport and child minding services, as well as associated medical costs, are also barriers to accessing contraception and health care services. Language barriers and an unfamiliar health care system were also stated to be inhibitors to accessing and utilisation of SRH services.

When you come to a foreign country and you feel you can't speak the language or you don't have a lot of money or you can't get on the train or you don't understand the systems, it's very isolating and difficult. (Advanced Practice Nurse)

Providing culturally sensitive staff members and interpreter services for health care clinics assists in reducing language barriers, however, they must also take into account the need for cultural competency relating to issues such as gender, eye-contact, tone of voice, body language and touch. Our key contact interviewee who works in the multicultural women's health sector stated, 'Cultural competency of both the health care professional and the interpreter is vital in making the client feel comfortable, safe and understood in a clinic environment', highlighting the need for cultural training for health care professionals and educators alike. Concerns about contraception use and side effects were another reported barrier to contraception use in the IDIs. It was noted that fears relating to contraception use can often be exacerbated by lack of contraception and SRH knowledge.

It's important that the client has a good understanding of how the contraception works within their bodies and if that's a healthy choice for them, both physically and also psychologically. (Advanced Practice Nurse)

\section{Effective Sexual and Reproductive Health Services: What do they entail?}

In the cultural context, male and female dynamics must be addressed to ensure effective and culturally sensitive SRH care education. This was a key theme reported in the IDIs. 
Effective SRH care education must include both male and female educators speaking with both men and women within the community. Speaking the language, knowing the culture and also being of the same gender as the group or individual you are speaking with, assists with the perceived credibility of the educator as well as creating an environment where people feel comfortable talking about sensitive issues. (Multicultural Women's Health Project Coordinator)

Whole community engagement, as a means of successful contraception and SRH provision strategy, was noted as a key factor to increasing knowledge and access to services for people within CALD communities. It was suggested that establishing trust within the community and rapport with the people can assist health care providers to more effectively meet and understand people's contraception and SRH needs.

It's about us going to them and introducing ourselves and letting ourselves been seen to be approachable and understanding. When it comes to sexual and reproductive health there's often fear or embarrassment or shame and meeting people within the community and being accessible in our manner can assist in overcoming these fears. (Advanced Practice Nurse)

\section{DISCUSSION}

A myriad of social, cultural, religious and economic factors were discussed as impacting the use of SRH services by our research participants. These contributing factors also play an inhibiting role in knowledge and understanding regarding contraception and SRH. At the 1994 Cairo International Convention on Population and Development (ICPD), the SRH needs of migrant populations including refugees and displaced people were officially acknowledged (Austin, Guy, Lee-Jones, McGinn, \& Schlecht, 2008; O’Heir, 2004; Sippel, 2008). The conference recognised that refugee, migrant and displaced populations in parts of the world where SRH care is already limited, faced increased threats to their reproductive health during times of humanitarian crisis and subsequent resettlement in new countries (Austin et al., 2008). 
Our research participants expressed lack of cultural competency and ineffective communication by health care providers as key barriers to positive health care seeking attitudes and the attainment of contraception and SRH knowledge. Lack of information about the Australian health care system and the perception that health beliefs, cultural values, and traditional health treatments are overlooked or disregarded by Australian health professionals, can cause dissatisfaction with treatment and result in patients being apprehensive to contact health providers (Benson \& Smith, 2007; Henderson \& Kendall, 2011).

Hebbani, Obijiofor and Bristed (2010) note that with parents living away from their homeland and culture, many Sudanese parents who have resettled in Australia wish to raise their children with the values and customs that they themselves were raised with. In the context of SRH education, this can present parents with many challenges as they seek to raise their children in a culture quite different to their own (Hebbani, Obijiofor \& Bristed, 2010).

This conflict of culture between young people and their parents in relation to SRH education within the home environment was highlighted as key area of concern for participants within the daughters' FGD. Lack of communication between parents and their children about safe sexual practices and behaviours, due to the topic being taboo, was reported as a barrier to contraception use and SRH knowledge for the younger generation. It was also noted in the IDIs that due to cultural or religious beliefs, children from CALD backgrounds can sometimes be excluded from classroom sexual health education at their parent's request. This can inhibit them from learning in school about safe sexual practices.

This intergenerational conflict was also noted as a concern young people have when attending health care clinics. Having older community members question them or discuss reasons for their attendance at clinics was reported as being a deterrent for young people seeking SRH knowledge and services. Cultural beliefs have been shown to impact on health seeking behaviour, knowledge and attitudes about SRH (Thomas et al., 2010).

Researchers have found that refugee and migrant populations represent a particularly vulnerable and at risk group for sexually transmissible infections and diseases (Asare, 2011; Tompkins, Smith, Jones \& Swindells, 2006). The perceived belief that an individual has about 
acquiring a disease can directly impact on an individual's decision to practice unsafe sexual behavior (Asare, 2011; Tompkins et al., 2006). Our research documented the reported perception that individuals within the Sudanese and Eritrean communities are engaging in risky sexual behaviors, such as having unprotected sex, under the assumption that sexually transmissible infections and diseases are not prevalent or do not exist in Australia. This belief is based on lack of SRH education, is compounded by cultural beliefs and attitudes towards contraception use, and the notion that all refugees and migrants entering Australia have medical clearance (Tompkins et al., 2006; WHO, 2008, 2011).

For effective contraception and SRH education to occur, community supported interventions are vital (Sheikh-Mohammed, Wood, Leask, \& Isaacs, 2006). Successful community based education projects have shown to be effective in addressing gender based inequalities as well as shifting social, religious and cultural barriers to SRH rights (UNFPA, 2008). Engagement with community leaders is an important step in sharing contraception and SRH knowledge as well as making community members aware of services available to them (Sheikh-Mohammed et al., 2006; UNFPA, 2008). Our study participants shared that both male and female educators and community health care providers, with an understanding of social and cultural issues impacting communities, can effectively and sensitively communicate SRH issues. Addressing language and health literacy barriers are also key components to increasing SRH knowledge within these communities.

There were a number of barriers to recruitment of participants; most significant of these was the reluctance for members of the communities to discuss contraception and SRH issues as these are considered taboo. Scheduling difficulties also compounded participant recruitment issues. While we gained rich and diverse data, it must be noted that due to the short time frame for completion of this study; the cross-sectional and exploratory nature of the study; and small numbers of participants, the results cannot be generalised. While we endeavoured to address language and cultural barriers by using interpreters during the mothers' FGD as well as a cultural interpreter to validate our findings, some bias may still have occurred.

\section{RECOMMENDATIONS}


An outcome of our research was participant proposed strategies and recommendations on how SRH interventions could be improved to address the health needs of people from CALD communities. An overarching theme that was noted in the participant suggested strategies was the recommendation that strategies to reduce language, cultural, and economic barriers to information and health services for CALD communities are crucial to the improvement of this population's health status (Henderson and Kendall, 2011; Murray et al., 2010; Sheik-Mohammed et al., 2006). We believe that the key to effective health care implementation is to create dialogue and knowledge exchange to enable health service providers to ask communities what they need. The following recommendations drawn from the FGDs are also supported by the literature:

- Establish links between CALD communities and health care professionals and services.

Collaboration between community leaders and elders, health service providers, and educators will assist in enabling whole community based and supported SRH education (Sheikh-Mohammed et al., 2006; UNFPA, 2008). Community based contact has the potential to create a holistic, culturally safe and sensitive environment in which people feel comfortable and supported.

- Provide people in CALD communities' access to culturally competent educators, health care professionals and translators.

Increasing cultural competency of educators, health care professionals and translators is paramount to effective and culturally sensitive education and service provision (Murray et al., 2010). By having educators and service providers such as community nurses and bilingual health workers who possess an understanding of the social, cultural, religious and gender based factors impacting contraceptive use and health care seeking attitudes, access to these services and knowledge of SRH will increase (Henderson et al., 2011).

- Provide translated health information that is accessible to different literacy levels to members of CALD communities.

Providing translated health information accessible to different literacy levels will enable a greater population of the community to gain knowledge on contraception and SRH as well as creating greater awareness of available services. The provision of SRH education material in their own language, as well as information regarding the health care system in their country of resettlement, 
would assist in reducing the culture shock many refugees and migrants experience when exposed to a new health care system (Benson \& Smith, 2007; Henderson \& Kendall, 2011).

- Provide culturally appropriate strategies for CALD parents to communicate with their children about SRH.

Empowering parents from CALD backgrounds with culturally appropriate strategies on how to communicate with their children about SRH will help enable the younger generation to gain better access to SRH knowledge and be better equipped to take control of their own SRH.

- Provide SRH education to people newly arrived in Australia as refugees and migrants during their resettlement phase.

During the resettlement phase holistic SRH education must address misconceptions people have regarding HIV/AIDS and STI prevalence in Australia. Policy makers, health care providers and community leaders must work together to establish accessible education as a point of first contact for these newly arrived people. This would also provide the opportunity to detail health and family planning services available to them in their local areas. Continual, ongoing education from the transition to the resettlement phase will work towards effectively meeting the refugee and migrant population's SRH needs and assist in integration into the wider community. This continual education and support also has the capacity to empower refugee and migrant populations to more effectively take control of their SRH rights.

\section{CONCLUSION}

This study has revealed the complexities of reproductive health and contraceptive use among a small group of Sudanese and Eritrean women in Brisbane, Australia. The World Health Organization (2010) states that unmet contraceptive and reproductive health needs are disproportionately higher amongst women from migrant, refugee and CALD backgrounds (WHO, 2010). Many of the social factors affecting women's health globally also affect migrant families, health programming as proposed in our recommendations needs to utilise methods that involve families and communities and include educational, and culturally appropriate components. Our proposed recommendations have the potential to assist migrant and refugee women in gaining greater knowledge and uptake of SRH care services in their country of resettlement. The disparities in health outcomes and health care seeking activities in women from CALD backgrounds in Australia highlights the need for more research on the SRH experiences and needs of these women (Henderson \& Kendall, 2011; Kelaher et al., 1999). The challenges identified in 
this pilot exploratory study can help facilitate reflection and discussion, and highlight the need to create the space and capacity to empower CALD women. We recognise that there are gaps in our knowledge, however, by respecting and listening to the voices of refugee and migrant women, we can develop holistic and inclusive strategies and health programs to improve SRH outcomes for this vulnerable yet resilient group of women. Understanding the needs and perspectives of refugee and migrant women is key to achieving effective and equitable SRH care for women within these communities. It is our hope that these recommendations will also inform better practices for SRH and GP services and provide greater insight into the health care needs of refugee and migrant communities not only in Australia but in other countries of resettlement globally.

\section{ACKNOWLEDGEMENTS}

We sincerely thank the women who gave their time to participate in this study. We are grateful to Dr Mohammed Ali from the Centre for International Health, Curtin University who reviewed an earlier version of this paper. We thank Odette Tewfik for her valued assistance in data collection and analysis; and Family Planning Queensland who facilitated the research. Finally we wish to thank the two anonymous reviewers who provided feedback on this article.

\section{REFERENCES}

Asare, M. (2011). Using the Health Belief Model and Acculturation to Predict Safer Sexual Behavior and Sexual Communication among African Immigrants. 3490840 (Ph.D.). University of Cincinnati, Ann Arbor. Retrieved from http://search.proquest.com/docview/917948307? accountid=10382

Austin, J., Guy, S., Lee-Jones, L., McGinn, T., \& Schlecht, J. (2008). Reproductive health: a right for refugees and internally displaced persons. Reproductive Health Matters, (16)31, 10-21. Retrieved from hhtp://www.rhm-elsevier.com

Australian Bureau of Statistics. (2008). Perspectives on Migrants, 2008 (No. 3416.0). Canberra, ACT: Author.

Australian Bureau of Statistics. (2011). Migration, Australia, 2009-10 (No. 3412.0). Canberra, ACT: Author.

Benson, J., \& Smith, M. (2007). Early health assessment of refugees. Australian Family Physician, 36(1), 41-3. Retrieved from http://search.proquest.com

Brisbane City Council (2012). Population and Dwelling Profile. Brisbane, QLD: Office of Economic and Statistical Research \& Queensland Treasury and Trade.

Burns, R. (2000). Part 3: Qualitative methods. In Introduction to Research Methods (4th ed.). (pp. 388-392). London: Sage Publications

Carroll, J., Epstein, R., Fiscella, K., Volpe, E., Diaz, K., \& Omar, S. (2007). Knowledge and beliefs about health promotion and preventive health care among Somali women in the United States. Health Care for Women International, 28(4), 360-380. doi:10.1080/07399330601179935

Deparment of Immigration and Citizenship. (2007). Population Flows: Immigration Aspects, 2006-2007. Canberra, ACT: Author.

Deparment of Immigration and Citizenship. (2011). Fact Sheet 60 - Australia's Refugee and Humanitarian Program. Canberra, ACT: Author. 
Harte, W., Childs, I. R. W., \& Hastings, P. A. (2011). African refugee communities in Southeast Queensland: Forces of concentration and dispersion. Australian Geographer, 42(3), 325-342. doi:10.1080/00049182.2011.595769

Hebbani, A., Obijiofor, L., \& Bristed, H. (2009). Generational differences faced by Sudanese refugee women settling in Australia. Intercultural Communication Studies, 18(1), 66-82. Retrieved from http://www.uri.edu/iaics/journal/index.php

Hebbani, A., Obijiofor, L., \& Bristed, H. (2010). Intercultural communication challenges confronting female Sudanese former refugees in Australia. Australasian Review of African Studies, 31(1), 37-61. Retrieved from http://www.afsaap.org.au

Henderson, S., \& Kendall, E. (2011). Culturally and linguistically diverse peoples knowledge of accessibility and utilisation of health services: Exploring the need for improvement in health service delivery. Australian Journal of Primary Health, 17(2), 195-201. doi: 10.1071/PY10065

Henderson, S., Kendall, E., \& See, L. (2011). The effectiveness of culturally appropriate interventions to manage or prevent chronic disease in culturally and linguistically diverse communities: A systematic literature review. Health and Social Care in the Community, 19(3), 225-249. doi:10.1111/j.1365-2524.2010.00972.x

Hinton, R., \& Earnest, J. (2009). Beyond risk factors to lived experiences: Young women's experiences of health in Papua New Guinea. Rural and Remote Health, 9(4), 1257-1257. Retrieved from hhtp://www.rrh.org.au

Hinton, R., \& Earnest, J. (2010). Stressors, coping, and social support among women in Papua New Guinea. Qualitative Health Research, 20(2), 224-38. doi:10.1177/1049732309357572

Implementing Best Practice Initiative(IPB). (2010). Family planning for health and development: actions for change. Meeting report. Geneva: Implementing Best Practices Initiative - WHO, USAID, UNFPA.

Jirojwong, S., \& Liamputtong, P. (2009). Population Health, Communities and Health Promotion. Melbourne, VIC: Oxford University Press.

Joyce, A., Earnest, J., de Mori, G., \& Silvagni, G. (2010). The experiences of students from refugee backgrounds at universities in Australia: Reflections on the social, emotional and practical challenges. Journal of Refugee Studies, 23(1), 82-97. doi:10.1093/jrs/feq001

Kelaher, M., Williams, G., \& Manderson, L. (1999). Towards evidence-based health promotion and service provision for new migrants to Australia. Ethnicity \& Health, 4(4), 305-313. doi:10.1080/13557859998074

Minichiello, V. (2008). Interview processes. In V. Minichiello, R. Aroni \& T. Hays (Eds.), In-depth interviewing: principles, techniques, analysis (pp. 77-124). New South Wales: Pearson Education Australia.

Mengisteab, K. (2010). Diversity Management in Africa: Findings from the African Peer Review mechanism and a Framework for Analysis and Policy Making. Geneva: United Nations. Economic Commission for Africa. Governance and Public Administration Division.

Mohajer, N., Bessarab, D., \& Earnest, J. (2009).There should be more help out here! A qualitative study of the needs of Aboriginal adolescents in rural Australia. Rural and Remote Health (9)

1137. Retrieved from: http://www.rrh.org.au

Murray, L., Windsor, C., Parker, E., \& Tewfik, O. (2010). The experiences of African women giving birth in Brisbane, Queensland. Health Care for Women International (31)458-472. doi:10.1080/07399330903548928

National Health and Medical Research Council. (2007). National Statement on Ethical Conduct in Human Research. Canberra: National Health and Medical Research Council and Australia's Vice Chancellors' Committee.

O'Heir, J. (2004). Pregnancy and childbirth care following conflict and displacement: Care for refugee women in low-resource settings. Journal of Midwifery \& Women's Health, 49(4), 14-18. doi: 10.1016/j.jmwh.2004.04.031

Psychosocial Working Group. (2003). Psychosocial interventions in complex emergencies: a conceptual framework. Edinburgh: Refugee Studies Centre, Queen Margaret University College.

Queensland Government. (2008). New futures: The Queensland government's engagement with African refugees. Brisbane, QLD: Queensland Government Department of Communities. 
Rodgers, B. L., \& Cowles, K. V. (1993). The qualitative research audit trail: A complex collection of documentation. Research in Nursing and Health, 16(3), 219-226. doi:10.1002/nur.4770160309

Ruppenthal, L., Tuck, J., \& Gagnon, A. J. (2005). Enhancing research with migrant women through focus groups. Western Journal of Nursing Research, 27(6), 735-754. doi: $10.1177 / 0193945905277157$

Sharts-Hopko, N. (2002). Assessing rigor in qualitative research. The Journal of the Association of Nurses in AIDS Care, 13(4), 84-86. Retrieved from http://search.proquest.com/docview/213289485?accountid=10382

Sheikh-Mohammed, M., MacIntyre, C. R., Wood, N. J., Leask, J., \& Isaacs, D. (2006). Barriers to access to health care for newly resettled sub-Saharan refugees in Australia. Medical Journal of Australia, 185(11), 594-7. Retrieved from http://search.proquest.com/docview/235704161?accountid=10382

Sippel, S. (2008). Achieving global sexual and reproductive health and rights. Human Rights, 35(1), 13-17. Retrieved from http://search.proquest.com

Strohschein, F., Merry, L., Thomas, J., \& Gagnon, A. (2010). Strengthening data quality in studies of migrants not fluent in host languages: a Canadian example with reproductive health questionnaires. Research in Nursing \& Health, 33(4), 369-379. doi:10.1002/nur.20390

Thomas, P. E., Beckmann, M., \& Gibbons, K. (2010). The effect of cultural and linguistic diversity on pregnancy outcome. The Australian \& New Zealand Journal of Obstetrics \& Gynaecology, 50(5), 419-422. doi:10.1111/j.1479-828X.2010.01210.x

Tompkins, M., Smith, L., Jones, K., \& Swindells, S. (2006). HIV education needs among Sudanese immigrants and refugees in the Midwestern United States. AIDS and Behavior, 10(3), 319-23. doi:10.1007/s10461-005-9060-8

United Nations High Commissioner for Refugees. (2013). UNHCR Global Report 2012. Retrieved August, 2013 from http://www.unhcr.org/gr12/index.xml

United Nations Population Fund. (2008). Female Genital Mutilation Free Village Model. Retrieved March, 2012 from http://egypt.unfpa.org/english/Project/b07886e7-5e99-4f97-a7baef6edf01461c

United Nations Population Fund. (2011). Making Reproductive Health Rights and Sexual and Reproductive Health a Reality For All. New York, NY: Author.

World Health Organization. (2008). Repositioning family planning: Guidelines for advocacy action. Geneva: World Health Organization, Regional Office for Africa, and the United States Agency for International Development (UNAIDS).

World Health Organization. (2010). Trends in maternal mortality: 1990 to 2008. Estimates developed by WHO, UNICEF, UNFPA and the World Bank. Geneva: Author.

World Health Organization. (2011). Fact sheet No 351: Family Planning. Retrieved March, 2012 from http://www.who.int/mediacentre/factsheets/fs351/en/index.html 